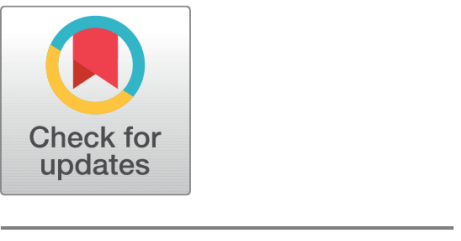

OPEN ACCESS

Received: 07.04.2021

Accepted: 11.05 .2021

Published: 13.09.2021

Citation: Mohiuddin M, Kannan R (2021) Alpha Power Transformed Aradhana Distributions, Its Properties and Applications. Indian Journal of Science and Technology 14(30): 2483-2493. https://doi.org/ 10.17485/JJST/v14i30.598

* Corresponding author. masmariam7@gmail.com

Funding: None

Competing Interests: None

Copyright: @ 2021 Mohiuddin \& Kannan. This is an open access article distributed under the terms of the Creative Commons Attribution License, which permits unrestricted use, distribution, and reproduction in any medium, provided the original author and source are credited.

Published By Indian Society for Education and Environment (iSee)

ISSN

Print: 0974-6846

Electronic: 0974-5645

\section{Alpha Power Transformed Aradhana Distributions, Its Properties and Applications}

\author{
Maryam Mohiuddin ${ }^{1 *}$, R Kannan ${ }^{2}$ \\ 1 Ph. D, Department of Statistics, Annamalai University, Tamil Nadu, 608002, India \\ 2 Professor, Department of Statistics, Annamalai University, Tamil Nadu, 608002, India
}

\section{Abstract}

Objectives: To introduce a new two-parameter lifetime distribution that will be more flexible in modeling real lifetime data over the existing common lifetime distributions. Methods: The new two-parameter lifetime distribution is generated by using the Alpha Power Transformed model developed by Mahdavi and Kundu. In this method, the probability density function and cumulative distribution function of Aradhana distribution are used as a base distribution for generating Alpha Power Transformed Aradhana Distribution. The probability density function and cumulative distribution function of the Aradhana distribution are substituted in the Alpha Power Transformed model to get the new and more flexible lifetime distribution for modeling real-life data. Findings: The authors reveal that the hazard rate of the Alpha Power Transformed Aradhana is increasing. They also found that the Alpha Power Transformed Aradhana distribution gives a much close fit than the two-parameter Aradhana distribution, Power Aradhana, Length Biased Garima, Exponential and Garima distribution. Novelty : In this study, a novel probability distribution is introduced. The Alpha Power Transformed Aradhana distribution is capable of modeling upside-down bathtub shaped hazard rates. The model is appropriate to fit the asymmetrical data that are not correctly fitted by other distributions. The said distribution can be applied to different fields like insurance, earthquake data for analysis, reliability etc.

Keywords: Reliability Analysis; Moments; Parameter Estimation

\section{Introduction}

The statistical analysis and fitting of lifetime data are important in all applied fields. The number of distributions of one and two parameters for modeling lifetime data is proposed by many researchers using different methods. The new lifetime model and regression model were discussed by Yousof et al. ${ }^{(1)}$ and studied the characterization of the model with the application. A new Two-Parameter lifetime model is discussed by Alizadeh et al. ${ }^{(2)}$ and studied the properties of the distribution with the application. A new generalization of the lifetime model based on the generalization of the half-normal 
distribution are introduced by Altun, Yousof et al. ${ }^{(3)}$ along with the properties of the model and regression models. Another generalization of the generalized exponential distribution along with properties and applications are developed by Zelibe et al. ${ }^{(4)}$. Para and Jan ${ }^{(5)}$ discussed the new lifetime distribution a generalization of Log-Logistic distribution for modeling medical data and obtained various properties of the model. Para and Jan ${ }^{(6)}$ discussed the three-parameter weighted Pareto Type II distribution and studied the properties with application in medical sciences.

Since the 1980s, researchers have diverted their attention from existing approaches to adding a parameter to existing distributions. The approach/method used by the researchers for generating new distributions were the beta-generated method, Transformed-Transformer method, Exponentiated generalized method etc. These methods were applied to the existing distributions for generating the new distributions which can overcome the lacking of the existing distributions.

Shanker ${ }^{(7)}$ introduced a new one-parameter distribution viz Aradhana distribution for modeling lifetime data. Let $\mathrm{Y}$ be the random variable having Aradhana distribution with parameter $\theta$, then the probability density function and cumulative distribution function of the distribution is given by

$$
\begin{gathered}
f(y, \theta)=\frac{\theta^{3}}{\theta^{2}+2 \theta+2}(1+y)^{2} e^{-\theta y}, \quad y>0, \theta>0 \\
F(y, \theta)=1-\left(1+\frac{\theta y(\theta y+2 \theta+2)}{\theta^{2}+2 \theta+2}\right) e^{-\theta y}, \quad y>0, \theta>0
\end{gathered}
$$

The Aradhana distribution is a combination of two components, exponential distribution $(\theta)$ and gamma distribution $(3, \theta)$. The author has discussed various mathematical properties of the distribution. The parameters of the Aradhana distribution are estimated by using the method of Maximum Likelihood estimation. In this article, the real lifetime data set is used to check the validity of the Aradhana distribution over one and two-parameter distributions. The authors found that the Aradhana distribution gives a much closer fit in modeling life-time data than Akash, Exponential, Shanker, Lindley distributions. Some of the modifications of the Aradhana distribution and their properties are introduced by Shanker.

Shanker and Welday ${ }^{(8)}$ introduced a generalized Aradhana distribution by using the concept of mixture models for modeling the data sets. The probability density function and cumulative distribution function of the two-parameter Aradhana distribution with parameters $\theta$ and $\alpha$, is given by

$$
\begin{aligned}
& f(y, \theta, \alpha)=\left(\frac{\theta^{3}}{\theta^{2}+2 \alpha \theta+2 \alpha^{2}}\right)(1+\alpha y)^{2} e^{-\theta y}, y>0, \theta>0, \alpha \geq 0 \\
& F(y, \theta, \alpha)=1-\left(1+\frac{\theta \alpha y(2 \theta+\theta \alpha y+2 \alpha)}{\theta^{2}+2 \theta \alpha x+2 \alpha^{2}}\right) e^{-\theta y}
\end{aligned}
$$

The said distribution is a combination of one-parameter exponential $(\theta)$ distribution, gamma $(3, \theta)$ distribution and gamma $(2, \theta)$ distribution. The Aradhana distribution is a special case of two-parameter Aradhana distribution. The properties of the distribution are discussed by the authors. The parameters of the two-parameter Aradhana distribution are estimated by using the method of Maximum Likelihood estimation. The authors used real lifetime data to verify the validity and flexibility of the Aradhana distribution on one and two-parameter distributions.

Quasi Aradhana distribution was proposed by Shanker and Shukla ${ }^{(9)}$ for modeling engineering data set. The probability density function and cumulative distribution function of the distribution having parameters $\theta$ and $\alpha$ is given by

$$
\begin{aligned}
& f(y, \theta, \alpha)=\left(\frac{\theta}{\alpha^{2}+2 \alpha+2}\right)(\alpha+\theta y)^{2} e^{-\theta y}, y>0, \theta>0, \alpha^{2}+2 \alpha+2>0 \\
& F(y, \theta, \alpha)=1-\left(1+\frac{\theta y(\theta y+2+2 \alpha)}{\alpha^{2}+2 \alpha+2}\right) e^{-\theta y}
\end{aligned}
$$

The Quasi Aradhana distribution is the mixture of exponential $(\theta)$, a gamma $(2, \theta)$, and gamma $(3, \theta)$ distribution. Various mathematical properties are discussed by the authors. The validation of the distribution is verified by using datasets and compared with gamma, Weibull, Lognormal, Aradhana, Lindley and exponential distributions.

A two-parameter power Aradhana distribution was discussed by Shanker and Shukla ${ }^{(10)}$. They have obtained the said distribution by the method of power transformation $Y=X^{\frac{1}{\alpha}}$.the probability density function and cumulative distribution 
function of the distribution is given by

$$
f(y, \theta, \alpha)=\left(\frac{\alpha \theta^{2}}{\theta^{2}+2 \theta+2}\right)(y)^{\alpha-1}\left(y^{2 \alpha}+2 y^{\alpha}+1\right) e^{-\theta y^{\alpha}}, y>0, \theta>0, \alpha>0
$$

The two-parameter power Aradhana distribution is a mixture of Weibull and generalized gamma distribution. The pdf of generalised gamma distribution introduced by Stacy $(1962)^{(11)}$ is given by

$$
f(y, \beta, \theta, \alpha)=\frac{\beta}{\theta \Gamma \alpha}\left(\frac{y}{\theta}\right)^{\alpha \beta-1} e^{-(y / \theta)^{\beta}}, y \geq 0, \beta, \theta>0, \alpha>0
$$

The purpose of this study is to develop another generalization of the Aradhana distribution called the Alpha Power Transformed Aradhana (APTAT) distribution using the APT model proposed by Mahdavi and Kundu ${ }^{(12)}$. The cdf of the APT family of distributions as

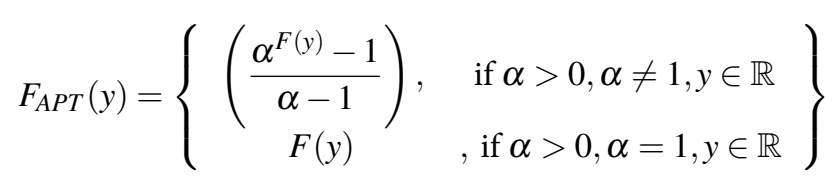

The corresponding probability density function as

$$
f_{A P T}(y)=\left\{\begin{array}{cc}
\left(\frac{\log \alpha}{\alpha-1}\right) f(y) \alpha^{F(y)}, & \text { if } \alpha>0, \alpha \neq 1, y \in \mathbb{R} \\
f(y) & \text { if } \alpha>0, \alpha=1, y \in \mathbb{R}
\end{array}\right\}
$$

Recently, some extensive work has been done on the Alpha Power family of distributions. Alpha power Transformed Frechet Distribution was introduced by Suleman et al. ${ }^{(13)}$ for modeling real-life data sets. Some of the statistical properties of the distribution such as quantile function, moments, mean residual life, generating function, entropy, stochastic ordering, etc. are discussed. The method of maximum likelihood estimation is used for estimating the parameters of the distribution.

The Alpha Power Transformed family, properties and Applications was developed by Mead, Cordeiro, Afify and AlMofleh $^{(14)}$. The author(s) reveal that the probability density function of the Alpha Power Transformation is the weighted function of the probability density function of the base distribution, here $\alpha^{G(x)}$ is the weight function for the model $g(x)$. The Statistical properties of the APT model are studied such as Linear representation of the model, Moments, Incomplete moments, moment generating function and order statistics. Further, the work was extended to introduce the Alpha Power Exponentiated Weibull distribution. The Alpha Power Exponentiated Weibull distribution uses the cdf of the Exponentiated Weibull distribution in the APT model. The Statistical properties of the APEW model is studied. This includes linear representation of the model, Moments, Incomplete moments, moment generating function and order statistics. A Simulation Study is also carried out to investigate the behaviour of the MLEs for different sample size.

Alpha Power Transformed Power Lindley distribution was suggested by Hassan ${ }^{(15)}$. Some statistical properties of the model are discussed such as quantile function, moments, probability weighted moments and stochastic ordering. The parameters of the model are estimates by maximum likelihood estimator and Maximum Product of Spacing Estimators.

Moreover, Ghosh ${ }^{(16)}$ introduced and studied properties of the Alpha Power Transformed Lindley distribution with application to Earthquake data. Alpha Power Transformed Inverse Power Lindley distribution was proposed by Nassar and $\operatorname{Kumar}^{(17)}$ and discussed properties of the model with the application. Alpha Power Transformed Quasi Lindley distribution was introduced by Patrick and Harrison ${ }^{(18)}$ and studied the properties of the model. Alpha Power Transformed Weibull-G was proposed by Golam Kibria ${ }^{(19)}$ with application to failure data. The distribution is generated by combining the two families of distributions APT-G family and Weibull-G family. The statistical properties of the APTW-G are derived and discussed. The APTW-G reduces to Alpha power transformed Weibull exponential distribution, Alpha power transformed Weibull Rayleigh distribution and Alpha power transformed Weibull Lindley distribution. Alpha Power Transformed Pareto distribution was introduced by Sakthivel ${ }^{(20)}$ and studied various properties of the distribution with the application. Alpha Power Transformed extended exponential distribution was proposed by Hassan ${ }^{(21)}$ and studied the properties of the model with the application. The alpha power extended exponential distribution reduces to alpha power exponential, alpha power Lindley, exponential, Lindley and gamma distribution. Alpha Power Transformed Inverse Lomax distribution was suggested by Hassan ${ }^{(1)}$ and studied the properties and different methods for estimating the parameters. 


\section{Alpha Power Transformed Aradhana (APTAD) Distribution}

Let $\mathrm{Y}$ be a random variable which is distributed as Alpha Power Transformed Aradhana distribution (APTAD) with scale parameter $\theta$ and shape parameter $\alpha$,and denoted by $\operatorname{APTAD}(\theta, \alpha)$. The pdf of the APTAD is obtained by substituting Equation (1) and Equation (2) in Equation (4) is given as

$$
f_{A P T}(y, \theta, \alpha)=\left(\frac{\log \alpha}{\alpha-1}\right) \frac{\theta^{3}}{\theta^{2}+2 \theta+2}(1+y)^{2} e^{-\theta y} \alpha^{1-\left(1+\frac{\theta y(\theta y+2 \theta+2)}{\theta^{2}+2 \theta+2}\right) e^{-\theta y}} y>0, \theta, \alpha>0, \alpha \neq 1
$$

The corresponding cumulative distribution of APTAD is obtained by substituting Equation (2) in Equation (3) as

$$
F_{A P T}(y, \theta, \alpha)=\frac{\alpha^{1-\left(1+\frac{\theta y(\theta y+2 \theta+2)}{\theta^{2}+2 \theta+2}\right) e^{-\theta y}-1}}{\alpha-1} \alpha>0, \alpha \neq 1
$$
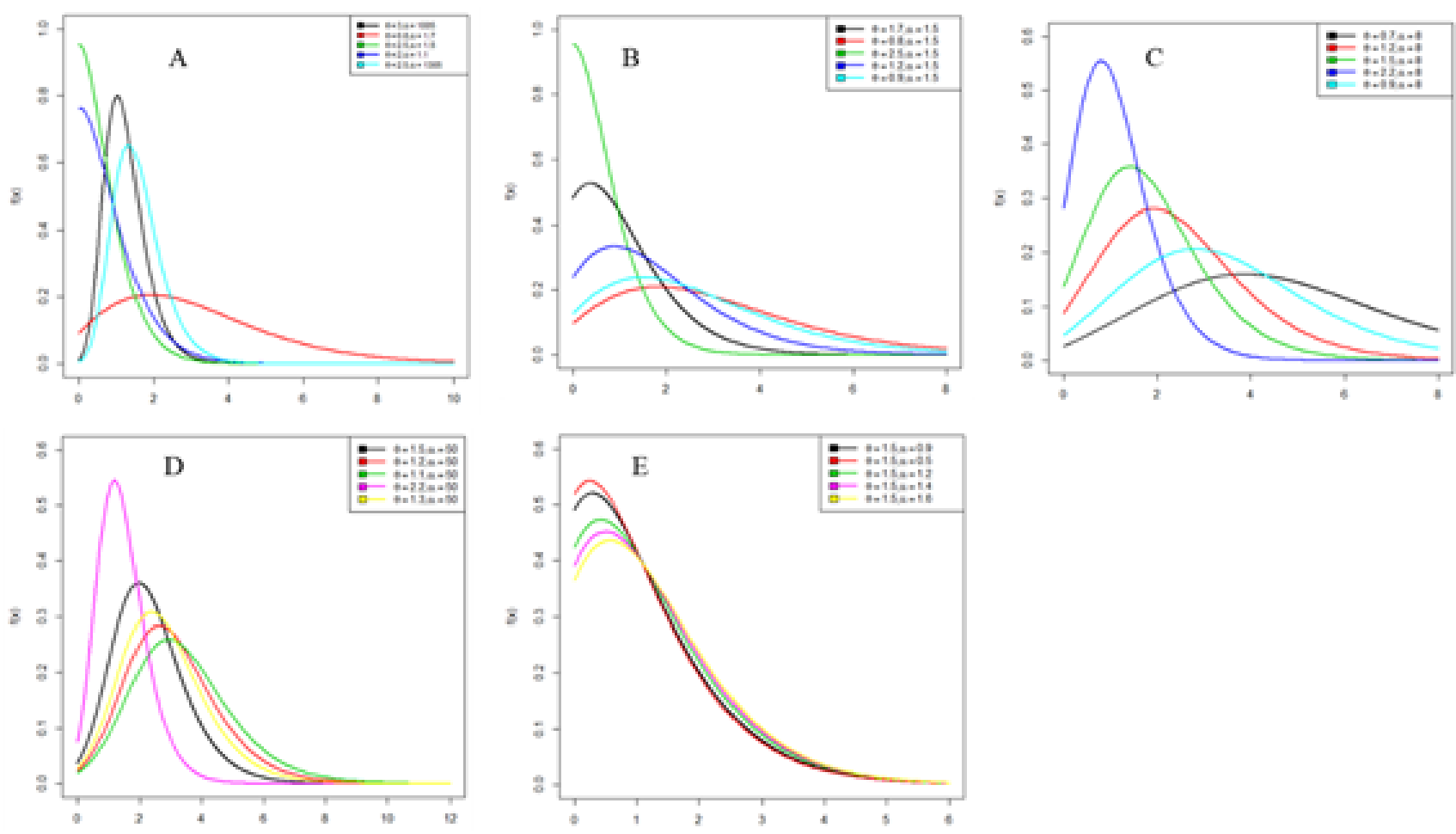

Fig 1. (A-E) pdf plot of Alpha Power Transformed Aradhana distribution 

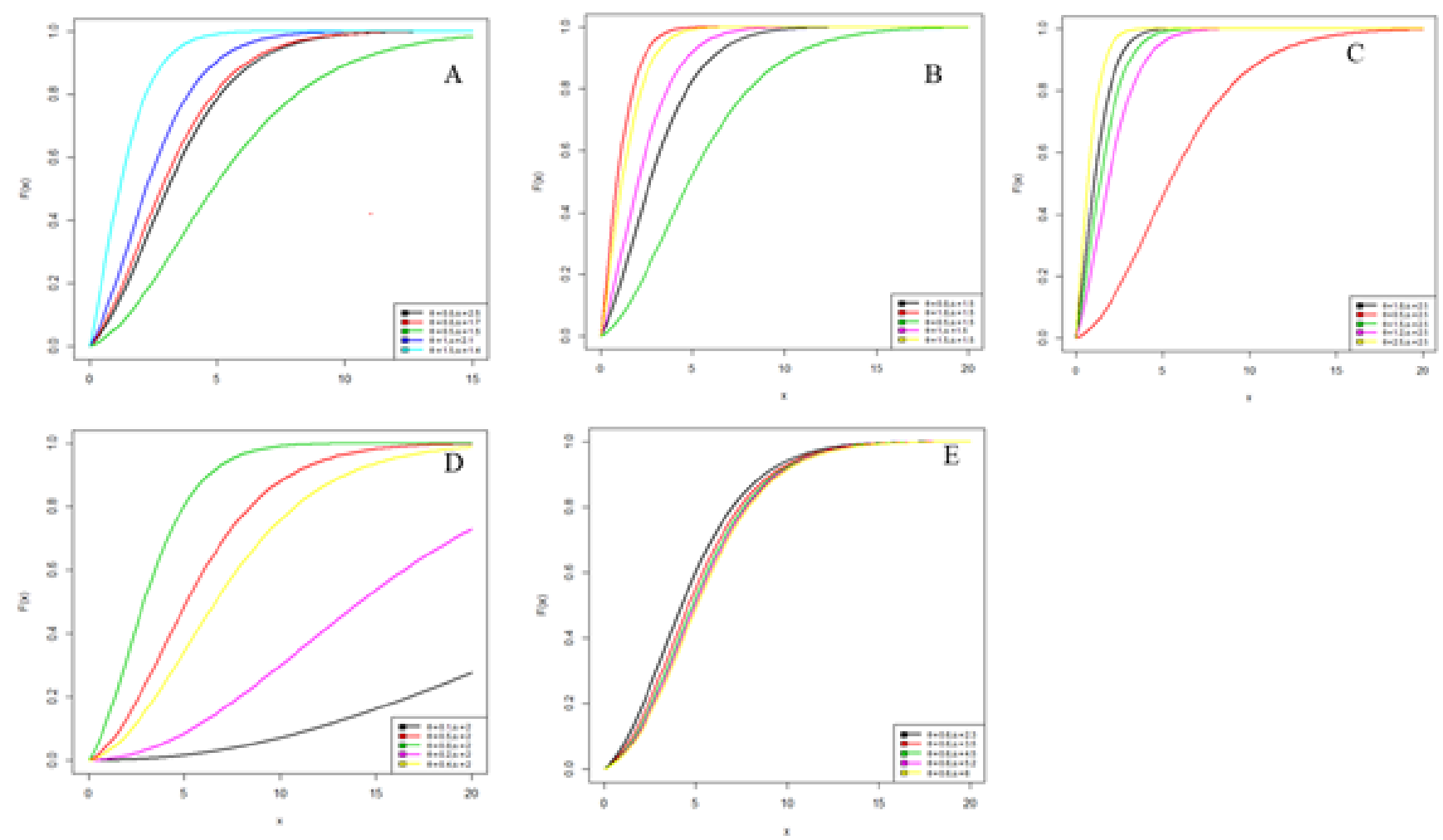

Fig 2. (A - E) cdf plot of Alpha Power Transformed Aradhana distribution

The reliability or survival function of the Alpha Power Transformed Aradhana distribution is given as

$$
S(y, \theta, \alpha)=\frac{\alpha-\alpha^{1-\left(1+\frac{\theta y(\theta y+2 \theta+2)}{\theta^{2}+2 \theta+2}\right) e^{-\theta y}}}{\alpha-1}
$$

The hazard function of the Alpha Power Transformed Aradhana distribution can be obtained as

$$
h(y, \theta, \alpha)=\frac{f(y, \theta, \alpha)}{S(y, \theta, \alpha)}
$$

Therefore,

$$
h(y, \theta, \alpha)=\frac{\log \alpha \theta^{3}(1+y)^{2} e^{-\theta y} \alpha^{1-\left(1+\frac{\theta y(\theta y+2 \theta+2)}{\theta^{2}+2 \theta+2}\right) e^{-\theta y}}}{\left(\alpha-\alpha^{1-\left(1+\frac{\theta y(\theta y+2 \theta+2)}{\theta^{2}+2 \theta+2}\right) e^{-\theta y}}\right)\left(\theta^{2}+2 \theta+2\right)}
$$




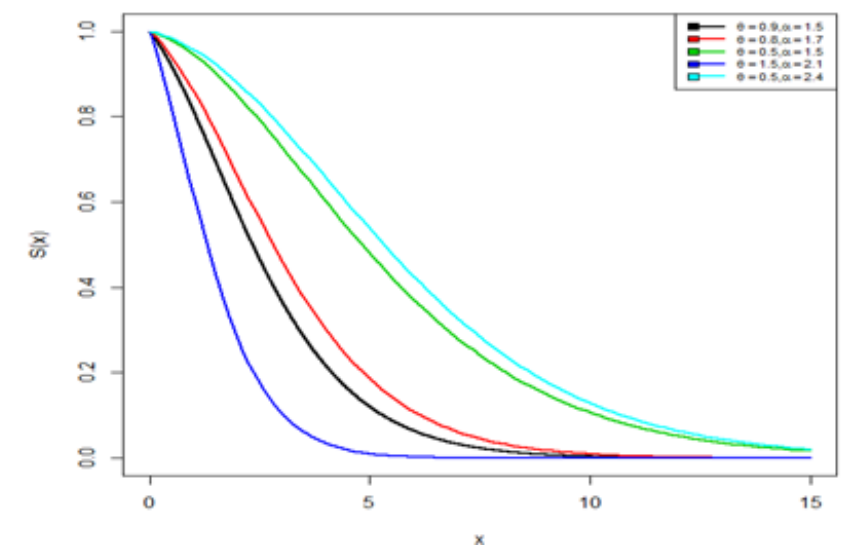

Fig 3. Survival plot of Alpha Power Transformed Aradhana distribution

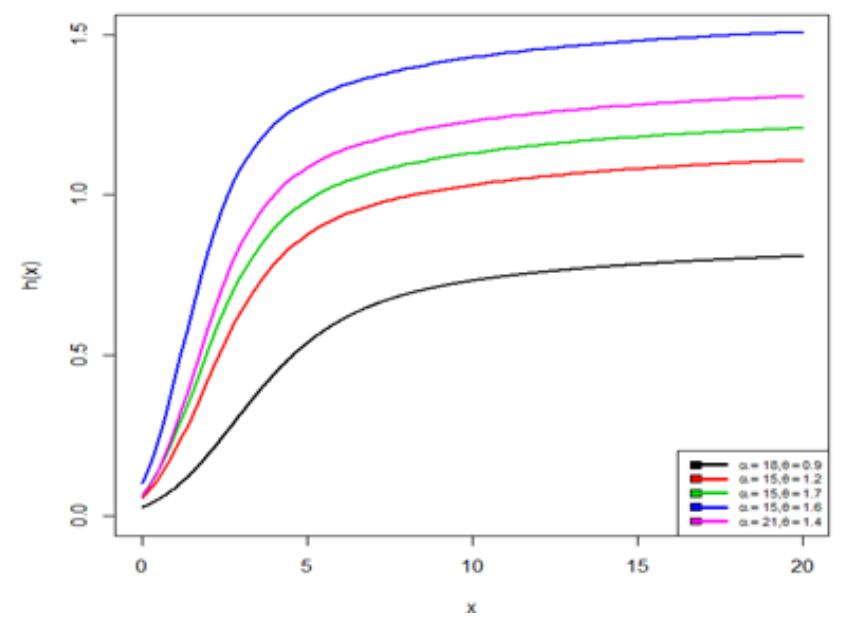

Fig 4. Hazard plot of Alpha Power transformed Aradhana distribution

\section{Statistical Properties}

In this section, some of the properties of the Alpha Power Transformed Aradhana distribution are discussed.

\section{Moments}

Let $\mathrm{Y}$ denote the random variable follows Alpha Power Transformed Aradhana distribution then $r^{\text {th }}$ order moment about origin of $\mu_{r}$ APTAD is

$$
\begin{gathered}
E\left(Y^{r}\right)=\int_{0}^{\infty} y^{r} f_{A P T}(y) d y \\
E\left(Y^{r}\right)=\int_{0}^{\infty} y^{r}\left(\frac{\log \alpha}{\alpha-1}\right)\left(\frac{\theta^{3}}{\theta^{2}+2 \theta+2}\right)(1+y)^{2} e^{-\theta y} \alpha{ }^{1-\left(1+\frac{\theta y(\theta y+2 \theta+2)}{\theta^{2}+2 \theta+2}\right) e^{-\theta y}} d y
\end{gathered}
$$


Using power series expansion to Equation (9),

$$
\alpha^{z}=\sum_{i=0}^{\infty} \frac{(\log \alpha)^{i}}{i !} z^{i}
$$

the Equation (9) becomes,

$$
E\left(Y^{r}\right)=\int_{0}^{\infty} y^{r} \sum_{i=0}^{\infty} \frac{(-\log \alpha)^{i}}{i !}\left(\left(1+\frac{\theta y(\theta y+2 \theta+2)}{\theta^{2}+2 \theta+2}\right) e^{-\theta y}\right)^{i}(1+y)^{2} e^{-\theta y}\left(\frac{\theta^{3}}{2+2 \theta+\theta^{2}}\right) d y
$$

We apply the binomial expansion $(1+x)^{n}=\sum_{i=0}^{n}\left(\begin{array}{c}n \\ i\end{array}\right) x^{i}$ to Equation (10), after simplification of the above Equation (10), we obtain the $r^{\text {th }}$ moment of the Alpha Power Transformed Aradhana distribution as

$$
\begin{aligned}
& E\left(Y^{r}\right)=\sum_{i=0}^{\infty} \sum_{j=0}^{i} \sum_{k=0}^{j} \sum_{l=0}^{k} \sum_{m=0}^{l}(-1)^{j}\left(\begin{array}{l}
l \\
m
\end{array}\right)\left(\begin{array}{c}
i \\
j
\end{array}\right)\left(\begin{array}{c}
j \\
k
\end{array}\right)\left(\begin{array}{c}
k \\
l
\end{array}\right)\left(\frac{(\log \alpha)^{k}}{k !}\right)\left(\frac{1}{\theta^{2}+2 \theta+2}\right)^{k+1} \theta^{k-m+4} \\
& \left\{\frac{(r+2 k-l) !}{(\theta(j+1))^{r+2 k-l+1}}+\frac{(r+2 k-l+1) !}{(\theta(j+1))^{r+2 k-l+2}}+\frac{(r+2 k-l+2) !}{(\theta(j+1))^{r+2 k-l+3}}\right\}
\end{aligned}
$$

Now we obtain the first four moments of the alpha power transformed Aradhana distribution by putting $r=1,2,3,4 \ldots$ in Equation (11) as

$$
\begin{aligned}
& E(Y)=\sum_{i=0}^{\infty} \sum_{j=0}^{i} \sum_{k=0}^{j} \sum_{l=0}^{k} \sum_{m=0}^{l}(-1)^{j}\left(\begin{array}{c}
l \\
m
\end{array}\right)\left(\begin{array}{c}
i \\
j
\end{array}\right)\left(\begin{array}{l}
j \\
k
\end{array}\right)\left(\begin{array}{c}
k \\
l
\end{array}\right)\left(\frac{(\log \alpha)^{k}}{k !}\right)\left(\frac{1}{\theta^{2}+2 \theta+2}\right)^{k+1} \theta^{k-m+4} \\
& \left(\frac{(1+2 k-l) !}{\theta^{2 k-l+2}(j+1)^{2 k-l+2}}+\frac{(2 k-l+3) !}{\theta^{2 k-l+3}(j+1)^{2 k-l+3}}+\frac{(2 k-l+4) !}{\theta^{2 k-l+4}(j+1)^{2 k-l+4}}\right) \\
& E\left(Y^{2}\right)=\sum_{i=0}^{\infty} \sum_{j=0}^{i} \sum_{k=0}^{j} \sum_{l=0}^{k} \sum_{m=0}^{l}(-1)^{j}\left(\begin{array}{c}
l \\
m
\end{array}\right)\left(\begin{array}{c}
i \\
j
\end{array}\right)\left(\begin{array}{c}
j \\
k
\end{array}\right)\left(\begin{array}{c}
k \\
l
\end{array}\right)\left(\frac{(\log \alpha)^{k}}{k !}\right)\left(\frac{1}{\theta^{2}+2 \theta+2}\right)^{k+1} \theta^{k-m+4} \\
& \left(\frac{(2+2 k-l) !}{\theta^{2 k-l+3}(j+1)^{2 k-l+3}}+\frac{(2 k-l+4) !}{\theta^{2 k-l+4}(j+1)^{2 k-l+4}}+\frac{(2 k-l+5) !}{\theta^{2 k-l+5}(j+1)^{2 k-l+5}}\right) \\
& E\left(Y^{3}\right)=\sum_{i=0}^{\infty} \sum_{j=0}^{i} \sum_{k=0}^{j} \sum_{l=0}^{k} \sum_{m=0}^{l}(-1)^{j}\left(\begin{array}{c}
l \\
m
\end{array}\right)\left(\begin{array}{c} 
\\
j
\end{array}\right)\left(\begin{array}{c}
j \\
k
\end{array}\right)\left(\begin{array}{c}
k \\
l
\end{array}\right)\left(\frac{(\log \alpha)^{k}}{k !}\right)\left(\frac{1}{\theta^{2}+2 \theta+2}\right)^{k+1} \theta^{k-m+4} \\
& \left(\frac{(3+2 k-l) !}{\theta^{2 k-l+4}(j+1)^{2 k-l+4}}+\frac{(2 k-l+5) !}{\theta^{2 k-l+5}(j+1)^{2 k-l+5}}+\frac{(2 k-l+6) !}{\theta^{2 k-l+6}(j+1)^{2 k-l+6}}\right) \\
& E\left(Y^{4}\right)=\sum_{i=0}^{\infty} \sum_{j=0}^{i} \sum_{k=0}^{j} \sum_{l=0}^{k} \sum_{m=0}^{l}(-1)^{j}\left(\begin{array}{c}
l \\
m
\end{array}\right)\left(\begin{array}{c}
i \\
j
\end{array}\right)\left(\begin{array}{c}
j \\
k
\end{array}\right)\left(\begin{array}{c}
k \\
l
\end{array}\right)\left(\frac{(\log \alpha)^{k}}{k !}\right)\left(\frac{1}{\theta^{2}+2 \theta+2}\right)^{k+1} \theta^{k-m+4} \\
& \left(\frac{(5+2 k-l) .}{\theta^{2 k-l+5}(j+1)^{2 k-l+5}}+\frac{(2 k-l+6) !}{\theta^{2 k-l+6}(j+1)^{2 k-l+6}}+\frac{(2 k-l+7) !}{\theta^{2 k-l+7}(j+1)^{2 k-l+7}}\right)
\end{aligned}
$$

\section{Moment Generating Function}

Let $Y$ be random variable follows Alpha Power Transformed Aradhana distribution, then the moment generating function (mgf) of $Y$ is obtained as

$$
\begin{aligned}
M_{Y}(t)=E\left(e^{t y}\right) & =\int_{0}^{\infty} e^{t y} f_{A P T}(y) d y \\
M_{Y}(t)=E\left(e^{t y}\right) & =\int_{0}^{\infty}\left(1+t y+\frac{(t y)^{2}}{2 !}+\ldots\right) f(y) d y \\
= & \int_{0}^{\infty} \sum_{r=0}^{\infty} \frac{t^{r}}{r !} y^{r} f(y) d y \\
= & \sum_{r=0}^{\infty} \frac{t^{r}}{r !} \mu_{r}^{\prime}
\end{aligned}
$$




$$
\begin{aligned}
& M_{T}(t)=\sum_{i=0}^{\infty} \sum_{j=0}^{i} \sum_{k=0}^{j} \sum_{l=0}^{k} \sum_{m=0}^{l}(-1)^{j} \sum_{r=0}^{\infty} \frac{t^{r}}{r !}\left(\begin{array}{l}
l \\
m
\end{array}\right)\left(\begin{array}{c}
i \\
j
\end{array}\right)\left(\begin{array}{c}
j \\
k
\end{array}\right)\left(\begin{array}{c}
k \\
l
\end{array}\right)\left(\frac{(\log \alpha)^{k}}{k !}\right)\left(\frac{1}{\theta^{2}+2 \theta+2}\right)^{k+1} \theta^{k-m+4} \\
& \left(\frac{(r+2 k-l) !}{\theta^{r+2 k-l+1}(j+1)^{r+2 k-l+1}}+\frac{(2 k-l+r+2) !}{\theta^{r+2 k-l+2}(j+1)^{r+2 k-l+2}}+\frac{(2 k-l+r+3) !}{\theta^{r+2 k-l+3}(j+1)^{r+2 k-l+3}}\right)
\end{aligned}
$$

Similarly, the characteristic function of Alpha Power Transformed Aradhana distribution can be obtained as

$$
\begin{aligned}
& \phi_{Y}(t)=M_{Y}(i t) \\
& M_{Y}(i t)=\sum_{i=0}^{\infty} \sum_{j=0}^{i} \sum_{k=0}^{j} \sum_{l=0}^{k} \sum_{r=0}^{\infty} \sum_{m=0}^{l}(-1)^{j} \frac{(i t)^{r}}{r !}\left(\begin{array}{l}
l \\
m
\end{array}\right)\left(\begin{array}{c}
i \\
j
\end{array}\right)\left(\begin{array}{c}
j \\
k
\end{array}\right)\left(\begin{array}{c}
k \\
l
\end{array}\right)\left(\frac{(\log \alpha)^{k}}{k !}\right)\left(\frac{1}{\theta^{2}+2 \theta+2}\right)^{k+1} \theta^{k-m+4} \\
& \left(\frac{(r+2 k-l) !}{\theta^{r+2 k-l+1}(j+1)^{r+2 k-l+1}}+\frac{(2 k-l+r+2) !}{\theta^{r+2 k-l+2}(j+1)^{r+2 k-l+2}}+\frac{(2 k-l+r+3) !}{\theta^{r+2 k-l+3}(j+1)^{r+2 k-l+3}}\right)
\end{aligned}
$$

\section{Order Statistics}

Let $Y_{(1)}, Y_{(2)}, Y_{(3)} \ldots Y_{(n)}$ be the order statistics of a random variable $Y_{1}, Y_{2}, Y_{3} . . Y_{n}$ drawn from the continuous population. $f(y)$ is the pdf while $F(y)$ is the cdf of the distribution, then the pdf of $r^{\text {th }}$ order statistic $Y_{(r)}$ is given by

$$
f_{Y(y)}(y)=\frac{n !}{(r-1) !(n-r) !} f(y)[F(y)]^{r-1}[1-F(y)]^{n-r}
$$

Inserting Equation (5) and Equation (6) in Equation (12), the pdf of $r^{\text {th }}$ order statistic $Y_{(r)}$ of the Alpha Power Aradhana distribution is given by

$$
\begin{aligned}
& f_{Y(r)}(x)=\frac{n !}{(r-1) !(n-r) !}\left(\frac{\log \alpha}{\alpha-1}\right)\left(\frac{\theta^{3}}{\theta^{2}+2 \theta+2}\right)(1+y)^{2} e^{-\theta y} \alpha^{1-\left(1+\frac{\theta y(\theta y+2 \theta+2)}{\theta^{2}+2 \theta+2}\right) e^{-\theta y}} \\
& {\left[\frac{\alpha^{1-\left(1+\frac{\theta y(\theta y+2 \theta+2)}{\theta^{2}+2 \theta+2}\right) e^{-\theta y}}-1}{\alpha-1}\right]^{r-1}\left[1-\frac{\alpha^{1-\left(1+\frac{\theta y(\theta y+2 \theta+2)}{\theta^{2}+2 \theta+2}\right) e^{-\theta y}}-1}{\alpha-1}\right]^{n-r}}
\end{aligned}
$$

Therefore the pdf of the higher-order statistic $Y_{(n)}$ can be obtained as

$$
f_{Y(y,)}(y)=n\left(\left(\frac{\log \alpha}{\alpha-1}\right)\left(\frac{\theta^{3}}{\theta^{2}+2 \theta+2}\right)(1+y)^{2} e^{-\theta y} \alpha^{1-\left(1+\frac{\theta y(\theta y+2 \theta+2)}{\theta^{2}+2 \theta+2}\right)^{-\theta y}}\right)\left(\frac{\alpha^{1-\left(1+\frac{\theta y(\theta y+2 \theta+2)}{\theta^{2}+2 \theta+2}\right)^{-\theta y}}-1}{\alpha-1}\right)^{n-1}
$$

and the pdf of the first order statistic $Y_{(1)}$ can be obtained as

$$
\begin{aligned}
& f_{Y(y,)}(y)=n\left(\left(\frac{\log \alpha}{\alpha-1}\right)\left(\left(\frac{\log \alpha}{\alpha-1}\right)\left(\frac{\theta^{3}}{\theta^{2}+2 \theta+2}\right)(1+y)^{2} e^{-\theta y} \alpha^{1-\left(1+\frac{\theta y(\theta y+2 \theta+2)}{\theta^{2}+2 \theta+2}\right) e^{-\theta y}}\right)\right) \\
& \left(1-\frac{\alpha^{1-\left(1+\frac{\theta y(\theta y+2 \theta+2)}{\theta^{2}+2 \theta+2}\right) e^{-\theta y}-1}}{\alpha-1}\right)
\end{aligned}
$$




\section{Parameter estimation}

Let $y_{1}, y, y_{3}, y_{4}, \ldots \ldots . . y_{n}$ be a random sample of size $\mathrm{n}$ from Alpha Power Transformed Aradhana Distribution. The likelihood function, L of Alpha Power Transformed Aradhana Distribution is given by

$$
\begin{aligned}
& L=\prod_{i=1}^{n}\left(\frac{\log \alpha}{\alpha-1}\right)\left(\frac{\theta^{3}}{\theta^{2}+2 \theta+2}\right)\left(1+y_{i}\right)^{2} e^{-\theta y_{i}} \alpha^{\left.1-1+\frac{\theta y_{i}\left(\theta y_{1}+2 \theta+2\right)}{\theta^{2}+2 \theta+2}\right)^{-\theta y_{i}}} \\
& L=\left(\frac{\log \alpha}{\alpha-1}\right)^{n}\left(\frac{\theta^{3}}{\theta^{2}+2 \theta+2}\right)^{n} \prod_{i=1}^{n}\left(1+y_{i}\right)^{2} e^{-\theta y_{i}} \alpha^{1-\left(1+\frac{\theta y_{i}\left(\theta y_{i}+2 \theta+2\right)}{\theta^{2}+2 \theta+2}\right)^{-\theta y_{i}}}
\end{aligned}
$$

Taking log on both sides of Equation (12), we get the log-likelihood function of the APTAD as

$$
\begin{aligned}
& \log L=n \log (\log \alpha)-n \log (\alpha-1)+3 n \log \theta-n \log \left(\theta^{2}+2 \theta+2\right)-\theta y_{i}+\sum_{i=1}^{n} \log \left(1+y_{i}\right)^{2} \\
& +\log \alpha \sum_{i=1}^{n}\left(1-\left(1+\frac{\theta y_{i}\left(\theta y_{i}+2 \theta+2\right)}{\theta^{2}+2 \theta+2}\right) e^{-\theta y_{i}}\right)
\end{aligned}
$$

By taking the first partial derivatives of the log-likelihood function with respect to the two parameters $(\alpha, \theta)$

$$
\begin{gathered}
\frac{\partial \log L}{\partial \alpha}=\left(\frac{n}{\alpha \log \alpha}\right)-\left(\frac{n}{\alpha-1}\right)+\frac{1}{\alpha} \sum_{i=1}^{n}\left(1-\left(1+\frac{\theta y_{i}\left(\theta y_{i}+2 \theta+2\right)}{\theta^{2}+2 \theta+2}\right) e^{-\theta y_{i}}\right) \\
\frac{\partial \log L}{\partial \alpha}=\left(\frac{3 n}{\theta}\right)-\left(\frac{n(2 \theta+2)}{\theta^{2}+2 \theta+2}\right)+\log \alpha \sum_{i=1}^{n}\left(1-\left(1+\frac{\theta y_{i}\left(\theta y_{i}+2 \theta+2\right)}{\theta^{2}+2 \theta+2}\right) e^{-\theta y_{i}}\right)
\end{gathered}
$$

The maximum likelihood estimates $(\hat{\alpha}, \hat{\theta})$ equations $\frac{\partial L}{\partial \alpha}=0, \frac{\partial L}{\partial \theta}=0$ The Equation (14) and Equation (15) cannot be solved as they both are in closed forms. So we compute the parameters of the Alpha Power Transformed Aradhana distribution using $R$ software $^{(22)}$. The optim function Nelder-Mead in R Statistical parameters.

\section{Application of Alpha Power Transformed Aradhana distribution}

The flexibility and performance of the Alpha Power Transformed Aradhana distribution are evaluated on competing models viz Aradhana distribution, Power Aradhana, Length Biased Garima, Exponential and Garima. Here, the distribution is fitted to two data sets, the performance of the distribution was compared with Two Parameter Aradhana distribution, Power Aradhana, Length Biased Garima, Exponential and Garima distributions for the data sets using Akaike Information Criterion (AIC), Bayesian Information Criterion (BIC), Akaike Information Criterion Corrected (AICC) and -2lnL. Distribution with the lowest AIC, BIC, AICC and -2InL is considered the most flexible and superior distribution for a given dataset. The results are presented in the tables below.

Data Set 1

The first data set taken by Nwikpe ${ }^{(23)}$ is the length of time (in years) that 81 randomly selected Nigerian graduates stayed without job before been employed by the universal basic education commission.

$2,5,7,5,6,7,7,6,6,9,9,6,6,7,5,4,5,2,9,8,5,9,6,6,7,2,8,3,6,6,2,8,5,7,4,5,6,8,8,9,3,7,6,2,6,8,9,7,6,6,9,5,9$, $5,5,5,3,9,8,6,6,6,7,9,4,4,6,9,7,8,8,9,4,6,3,5,4,7,6,6,5$.

\section{Data Set 2}

The second data set is the strength data of glass of the aircraft window given by Fuller ${ }^{(24)}$. The data set is given below $18.83,20.80,21.657,23.03,23.23,24.05,24.321,25.50,25.52,25.80,26.69,26.77,26.78,27.05,27.67,9.90,31.11,33.20,33.73$, $33.76,33.89,34.76,35.75,35.91,36.98,37.08,37.09,39.58,44.045,45.29,45.381$. 
Table 1. Goodness of fit the Alpha Power Transformed Aradhana distribution for data set 1

\begin{tabular}{|c|c|c|c|c|c|c|}
\hline Model & ML Estimates & Standard Deviation & AIC & BIC & AICC & $-2 \ln \mathrm{L}$ \\
\hline Two Parameter Aradhana & $\widehat{\theta}=0.3821 \widehat{\alpha}=0.5528$ & 0.03960 .2254 & 368.6978 & 373.4867 & 369.0095 & 364.6978 \\
\hline $\begin{array}{l}\text { Alpha power transformed } \\
\text { Aradhana Distribution }\end{array}$ & $\widehat{\theta}=0.7793 \widehat{\alpha}=1.0856$ & 0.04811 .4801 & 351.5837 & 356.3726 & 351.8954 & 347.5838 \\
\hline LB Garima & $\widehat{\theta}=0.4287$ & 0.0299 & 394.6677 & 397.0622 & 394.7183 & 392.6677 \\
\hline Exponential & $\widehat{\theta}=0.1639$ & 0.0182 & 456.91 & 459.3044 & 456.9606 & 454.9100 \\
\hline Garima & $\widehat{\theta}=0.2484$ & 0.0234 & 442.3109 & 444.7053 & 442.3615 & 440.3109 \\
\hline $\begin{array}{l}\text { Power Aradhana Distribu- } \\
\text { tion }\end{array}$ & $\widehat{\theta}=0.2202 \widehat{\alpha}=0.1907$ & 0.02270 .0189 & 1427.962 & 1432.751 & 1428.273 & 1423.962 \\
\hline
\end{tabular}

Table 2. Goodness of fit the Alpha Power Transformed Aradhana distribution for data set 2

\begin{tabular}{|c|c|c|c|c|c|c|}
\hline Model & ML Estimates & Standard Deviation & AIC & $\mathrm{BIC}$ & AICC & $-2 \ln \mathrm{L}$ \\
\hline Two Parameter Aradhana & $\widehat{\theta}=0.0794 \widehat{\alpha}=0.0996$ & 0.01110 .0433 & 231.6854 & 234.5534 & 232.5743 & 227.6855 \\
\hline $\begin{array}{l}\text { Alpha Power Transformed } \\
\text { Aradhana }\end{array}$ & $\widehat{\theta}=0.1975 \widehat{\alpha}=1.9082$ & 4.93461 .6787 & 213.9172 & 216.7852 & 214.8061 & 209.9173 \\
\hline LB Garima & $\widehat{\theta}=0.0877$ & 0.0099 & 248.5801 & 250.0141 & 248.7180 & 246.5801 \\
\hline Exponential & $\widehat{\theta}=0.0324$ & 0.0058 & 276.5289 & 277.9629 & 276.6668 & 274.5289 \\
\hline Garima & $\widehat{\theta}=0.0515$ & 0.0079 & 269.2780 & 270.7120 & 269.4159 & 267.2780 \\
\hline $\begin{array}{l}\text { Power Aradhana Distribu- } \\
\text { tion }\end{array}$ & $\widehat{\theta}=0.2170 \widehat{\alpha}=0.1015$ & 0.03650 .0165 & 691.8346 & 694.7025 & 692.7234 & 687.8346 \\
\hline
\end{tabular}

\section{Conclusion}

In this paper, a novel probability distribution is introduced. The new distribution is a two- parameter distribution, Alpha Power Transformed Aradhana distribution. Some of the properties are derived and discussed like moments, order statistics, reliability analysis, and hazard rate. The method of maximum likelihood estimation is used for determining the parameters. The performance of the new model is determined by fitting it to two real-life data using the goodness of fit criteria such as AIC, BIC, AICC and $2 \log$ L. It is found that Alpha Power Transformed Aradhana distribution gives a better fit to the data set as compared with exponential, Power Aradhana, Length Biased Garima, Exponential and Garima distributions. Further, The Alpha Power Transformed can be applied to various areas. The Alpha Power Transformed Aradhana distribution may suitable for most of the lifetime data and provides better outcomes than other well-known distributions.

\section{References}

1) Yousof HM, Altun E, Rasekhi M, Alizadeh M, Hamedani G, New AMM. Lifetime Model with Regression Models, Characterizations and Applications. Communications in Statistics-Simulation and Computation. 2019;48:264-286. Available from: https://doi.org/10.1186/s40488-018-0089-4.

2) Alizadeh M, Emadi M, Doostparast M. A New Two-Parameter Lifetime Distribution: Properties, Applications and Different Method of Estimations. Statistics, Optimization \& Information Computing. 2019;7:291-310. Available from: https://doi.org/10.19139/soic.v7i2.653.

3) Altun E, Yousof H, Hamedani G. A new generalization of generalized half-normal distribution: properties and regression models. Journal of Statistical Distributions and Applications. 2018;5(7). Available from: https://doi.org/10.1186/s40488-018-0089-4.

4) Eghwerido JT, Zelibe SC, Ekuma-Okereke E, Efe-Eyefia E. On the extented new generalized exponential distribution: Properties and Applications. FUPRE Journal of Scientific and Industrial Research. 2019;3(1):112-122.

5) Para BA, R JT. A new generalized version of Log-logistic distribution with applications in medical sciences and other applied fields. International Journal of Modeling, Simulation, and Scientific Computing. 2018;9(5). doi:10.1142/S1793962318500435.

6) Para BA, R JT. On three Parameter Weighted Pareto Type II Distribution: Properties and Applications in. Medical Sciences Applied Mathematics and Information Sciences Letters. 2018;6(1):13-26. Available from: http://dx.doi.org/10.18576/amisl/060103.

7) Shanker R. Aradhana Distribution and Its Applications. International Journal of Statistics and Applications. 2016;6(1):23-34. Available from: https://doi: 10.5923/j.statistics.20160601.04.

8) Shanker R, Daneil WA. Generalized Aradhana Distribution with Properties and Applications. International Journal of Biometrics and Biostatistics. 2018;7(4):374-385.

9) Shanker R, Shukla KK, Quasi A. Aradhana Distribution with Properties and Application. International Journal of Statistics and Systems. 2018;13(1):61-80. Available from: https://www.ripublication.com/ijss18/ijssv13n1_07.pdf.

10) Shanker R, and KKS. A Two Power Aradhana Distribution with Properties and Application. Indian Journal of Industrial and Applied Mathematics. 2018;9(2):210-220. 
11) Stacy EW. A generalization of the gamma distribution. Annals of Mathematical Statistics. 1962;33(3):1187-1192.

12) Mahdavi A, Kundu D. A new method for generating distributions with an application to exponential distribution. Communications in Statistics-Theory and Methods. 2017;46(13):6543-6557. Available from: https://www.tandfonline.com/doi/abs/10.1080/03610926.2015.1130839.

13) Nasiru S, Peter N. Alpha Power Transformed Frechet Distribution. International Journal Applied Mathematics \& Information Sciences. 2019;13(1):129-141. Available from: http://dx.doi.org/10.18576/amis/.

14) Mead ME, Cordeiro GM, Afify AZ, Al-Mofleh H. The Alpha Power Transformation Family: Properties and Applications. Pakistan Journal of Statistics and Operation research. 2019;15(3):525-545.

15) Hassan AS, Elgarhy M, Mohamd RE, Alrajhi S. Alpha Power Transformed Power Lindley distribution. Journal of Probability and Statistics. 2019;p. Article ID 8024769. Available from: https://doi.org/10.1155/2019/8024769.

16) Dey S, Ghosh I, Kumar D. Alpha-Power Transformed Lindley Distribution: Properties and Associated Inference with Application to Earthquake Data. Annals of Data Science. 2019;6:623-650. Available from: https://link.springer.com/article/10.1007/s40745-018-0163-2.

17) Dey S, Nassar M, Kumar D. Alpha power transformed inverse Lindley distribution: A distribution with an upside-down bathtub-shaped hazard function. Journal of Computational and Applied Mathematics. 2018;348:130-145.

18) Patrick U, Harrison E. Alpha Power Transformed Quasi Lindley distribution. International Journal of Advanced Statistics and Probability. 2021;9(1):6-7. doi:10.14419/ijasp.v9i1.31208.

19) Elbatal I, Elgarhy M, Kibria BMG. Alpha Power Transformed Weibull-G Family of Distributions. Journal of Statistical Theory and Applications. 2021;p. 130-145. Available from: https://doi.org/10.1016/j.cam.2018.03.037.

20) Sakthivel KM. Alpha power transformed Pareto distribution and its properties with application. Journal of Mathematik. 2020. doi:10.26637/MJM0S20/0011.

21) Ramadan A, Eldin Z, Haq MAU, Hashmi S, Elsehety M. Alpha Power Transformed Inverse Lomax Distribution with Different Methods of Estimation and Applications. Journal of Probability and Statistics. 2020;(1860813). Available from: https://doi.org/10.1155/2020/1860813.

22) Team RC. R, A Language and Environment for Statistical Computing. R Foundation for Statistical Computing. 2019.

23) Barinaandaa NJ, Isaac ED, Emeka A. Nwikpe Probability Distribution, Statistical Properties and Goodness of Fit. Asian Journal of Probability and Statistics. 2021;p. 52-61. Available from: https://doi.org/10.9734/ajpas/2021/v11i130260.

24) Fuller EJ, Frieman S, Quinn J, Quinn G, Carter W. Fracture mechanics approach to the design of glass aircraft windows. A case study. Proceedings SPIE. 1994;2286:419-430. Available from: https://doi.org/10.1117/12.187363. 